\title{
Analyses et comptes rendus
}

BeBEy, Kidi (dossier coordonné par). — Francis Bebey, l'homme-orchestre. Paris, L'Harmattan, 2002, 127 p. (Africultures, nº 49, juin).

Cet hommage collectif à la carrière de Francis Bebey est tout à fait touchant. Écrit par des collaborateurs qui le connaissent bien, vous découvrirez un artiste camerounais aux mille visages.

Ceux et celles qui connaissent le parcours de Francis Bebey savent que cet artiste est multiforme, c'est-à-dire qu'il était écrivain, nouvelliste, poète, romancier, conteur et finalement musicien. Cet «homme-orchestre », si on peut le définir ainsi pour refléter l'extraordinaire capacité de création de cet homme, nous sera donc raconté par l'entremise de ses œuvres et de ses proches. Vous lirez Toups Bebey, fils de Francis, qui nous fait découvrir l'homme, le père. D'ailleurs, ce témoignage nous amènera directement au langage musical, à celui qui n'a pas besoin de mots, mais plutôt d'émotions et d'intentions. Cette complicité père-fils, à elle seule, saura bien vous convaincre de cette énorme profondeur qui caractérise si bien l'homme qu'était Francis Bebey. D'autres collaborateurs, tels que Kidi Bebey, Bernard Magnier, Sophie Ekoué, Frank Tenaille et Gérald Arnaud, pour ne nommer que ceux-ci, nous ferons découvrir, chacun à leur façon, une autre facette de ce fascinant personnage.

Lorsque j'ai lu, pour la première fois, ces quelques pages concernant le parcours personnel de Francis Bebey, je n'ai pu m'empêcher de penser que le processus de «création » d'une œuvre n'a pas besoin de frontière disciplinaire, qu'il n'est pas régi par un instrument quelconque, une parole ou une préférence médiumnique. Ici, il y a peut-être une différence entre le musicien et l'artiste. Entre celui qui s'exprime par le truchement d'un instrument et celui qui décide d'utiliser son âme comme moyen d'expression de ce qu'il est vraiment, c'està-dire tout simplement un homme convaincu que certaines valeurs doivent être régies par le cœur, et non par l'isolement et le déni.

Pendant la rédaction de ce texte, je n'ai pu m'empêcher de penser à Manu Dibango, Anne-Marie N'Zié, Eboa Lottin ainsi qu'à certains autres individus qui ont engagé le Cameroun dans un processus de création important. En écoutant chanter Youssou N'Dour, je m'imagine le sourire et entend cette phrase de Bebey : «Si je n'étais pas noir, comment saurais-tu que tu es blanche... ?» J'espère que ce livre vous fera le même effet...

Marcel SAVARD

Cahiers d'Études africaines, 168, XLII-4, 2002, pp. 875-886. 
Chitando, Ezra. - Singing Culture. A Study of Gospel Music in Zimbabwe. Uppsala, Nordiska Afrikainstitutet, 2002, 105 p., discography, bibl. (« Research Report »121).

Depuis le début des années 1990, le gospel a conquis la scène et l'industrie musicales zimbabwéennes. Les cassettes des différents groupes et chanteurs individuels regroupés sous l'appellation «gospel » inondent le marché (des flea markets, sortes de marchés aux puces en zone urbaine, aux grands magasins), les programmes de radio et de télévision spécialisés sont très nombreux et suscitent l'enthousiasme du public, les morceaux sont joués tant chez les particuliers que dans la rue, les commuter buses et les boîtes de nuit, et les affiches de concert tapissent les murs des grandes villes.

Ezra Chitando, conférencier à l'université du Zimbabwe et auteur de nombreux articles en histoire et phénoménologie des religions, se propose donc d'envisager en soi ce phénomène populaire, et plus largement comme un exemple des productions culturelles de l'Afrique contemporaine.

Le gospel au Zimbabwe est défini comme «an artistic product emerging from cultural workers who are influenced by the Christian cumulative tradition » (p. 14) provenant d'artistes qui utilisent différents styles musicaux et instruments pour communiquer des thèmes chrétiens, représentant ainsi « the creative interplay between traditional musical performances, Christian influences, and global music trends » (p. 15). Afin d'expliquer le succès actuel de ce type de musique, l'auteur adopte une méthode pluridisciplinaire. Une approche historique "plante le décor » à l'ensemble de l'étude. Des outils d'analyse sociologique permettent de marquer le lien entre le contexte social et l'utilisation des thèmes chrétiens dans la culture populaire au Zimbabwe. Enfin, une interprétation de type phénoménologique aide à clarifier les aspects religieux du fait culturel étudié et à mettre en évidence les mélanges à l'œuvre dans les productions culturelles africaines contemporaines en général. Ezra Chitando aborde donc le gospel comme une émanation religieuse à mettre en lien avec l'ensemble du contexte historique, économique, culturel et politique qui a vu son émergence sous la colonisation puis son affirmation durant la dernière décennie du $\mathrm{Xx}^{\mathrm{e}}$ siècle.

L'ouvrage s'articule en 7 parties. Les deux premières sont consacrées à la présentation du sujet de recherche, des conditions dans lesquelles elle a été effectuée, et de la démarche qui a présidé à sa réalisation. Ceci confère à Singing Culture un aspect très « universitaire », surprenant au premier abord, mais vient démontrer que pour l'auteur, c'est autant son sujet que la manière dont il l'envisage qui est important. Il propose ensuite un résumé de l'histoire de la musique au Zimbabwe. Mise en lien avec l'histoire du pays, cette partie permet à l'auteur d'affirmer l'importance de la contextualisation de son objet de recherche. Et de déjouer ainsi de nombreuses idées reçues, quant à, notamment, l'immobilisme culturel précolonial des cultures africaines, et à la ténuité du lien entre les cultures contemporaines et traditionnelles: bien avant la colonisation anglaise, les Shona, le principal groupe culturel au Zimbabwe, avaient pratiqué de nombreux échanges commerciaux, avec des pays arabes et asiatiques entre autres, qui débouchèrent toujours sur des emprunts culturels. Par ailleurs, on retrouve, dans tous les styles de musique coloniaux et postcoloniaux produits au Zimbabwe, des traces de la culture Shona dite «traditionnelle ». Le gospel utilise ainsi des techniques vocales, des instruments, des rythmes et des schémas 
mélodiques « ancestraux », à côté d'influences diverses puisées dans le christianisme et dans la culture occidentale contemporaine (principalement nordaméricaine). Ezra Chitando retrace rapidement à cette occasion l'histoire des religions chrétiennes au Zimbabwe et de leurs dérivées plus récentes, actuellement très ancrées (selon une étude relatée dans l'ouvrage, plus de $65 \%$ de la population se déclarent de foi chrétienne), et en explicite les conséquences actuelles sur l'ethos de la population (permettant à son tour d'expliquer en partie la grande popularité des thèmes évoqués dans le gospel).

Nous revenons ensuite plus spécifiquement au sujet, avec une analyse de la progression du gospel dans les années 1970, puis de son explosion populaire et commerciale depuis le début des années 1990. Là encore, les événements politiques servent de toile de fond à cette progression d'un «nouveau » style musical sur la scène zimbabwéenne durant les 30 dernières années. L'UDI, tout d'abord (Unilateral Declaration of Independence, en 1962, autrement appelée l'indépendance blanche d'Ian Smith, qui fait entrer le Zimbabwe dans une longue période d'isolement commercial et diplomatique), qui a pour résultat, concernant uniquement la scène artistique et culturelle, d'encourager les talents locaux, et permet donc à de nombreux musiciens de s'affirmer sur le marché national ; la guerre de libération, ensuite, durant laquelle les nationalistes utilisent des chants inspirés d'hymnes religieux populaires; puis l'indépendance, suivie d'une décennie enthousiaste passée à glorifier les vétérans du conflit, l'émergence du nouveau pays, son nouveau gouvernement, et son chef d'État, Robert Mugabe ; enfin, la chute, la décadence politique et économique d'un pays qui représentait un grand espoir pour l'Afrique australe, auxquelles vient s'ajouter le sida. Une décennie difficile pour la majorité des Zimbabwéens, mais qui voit, pour différentes raisons historiques, sociologiques et culturelles, le gospel conquérir la scène populaire et le marché de la musique. Les chanteurs de gospel, comme la plupart des autres musiciens du pays, se font les porteparoles du peuple et dénoncent les erreurs et les échecs du gouvernement, affirmant pour chacun le droit à une vie décente, à travers ce que Chitando appelle les protest songs. Preuve que même à cette période de lutte quotidienne, l'innovation et la créativité continuent de caractériser la scène musicale zimbabwéenne, nourrissant une industrie musicale très florissante, paradoxalement avec la situation économique générale. Ce succès est largement relayé et supporté par les médias zimbabwéens, notamment à travers la radio et la télévision, qui consacrent aux musiques à thèmes chrétiens de nombreux programmes, mobilisant une forte audience.

L'auteur tente également de mesurer et d'analyser l'importance économique du gospel, et fait état de l'espace d'expression qu'offre cette catégorie musicale à des groupes muselés socialement.

Petit à petit, donc, le gospel au Zimbabwe a quitté les édifices religieux, pour entrer sur l'espace public. Cela s'est opéré de manière tout d'abord indirecte, les chants de lutte de la guerre de libération (les chimurenga songs) se calquant sur des hymnes religieux et les premiers morceaux de gospel. Aujourd'hui adopté par l'ensemble de la population zimbabwéenne, il se distingue des différentes Églises d'où il provient initialement pour devenir partie intégrante de la scène artistique et de l'industrie musicale nationale, s'exportant même de plus en plus (dans la région, mais aussi vers les États-Unis, entre autres).

Cet ouvrage constitue donc en premier lieu l'explication d'un phénomène musical contemporain, puisant sa source dans l'histoire du pays et dans des interactions actuelles avec les cultures occidentales. 
Mais, ce qui nous semble mériter tout particulièrement l'attention, c'est qu'à travers l'étude du gospel, l'auteur s'offre la possibilité, grâce à différents outils théoriques, de retracer tout le contexte qui a amené la naissance et l'affirmation de cet aspect contemporain de la culture zimbabwéenne. Les thèmes abordés à ce titre sont nombreux, on citera entre autres : l'implantation des missions dans le Zimbabwe précolonial, l'évolution du christianisme et son impact sur la société et les pratiques culturelles, notamment traditionnelles, les problèmes politiques et économiques se posant depuis l'indépendance, la domination de modèles culturels hérités de la colonisation et renforcés par le contexte mondial de globalisation, etc. Le rôle de la femme dans la société shona, puis rhodésienne et dans le cadre de l'urbanisation croissante du Zimbabwe contemporain, et plus spécifiquement son rôle dans la production de la musique, apparaît de manière récurrente, dans l'ensemble de l'ouvrage. Cette démarche revient au final à dresser une sorte de portrait contemporain de la société zimbabwéenne à travers une de ses productions artistiques et culturelles.

Il nous apparaît donc que Singing Culture constitue une sorte de revendication et d'appel. Revendication tout d'abord car les cultures africaines sont en perpétuel changement. Au contemporain, elles sont particulièrement créatives, se nourrissant tant de racines traditionnelles que de diverses influences résultant de leur rencontre avec d'autres cultures. Les produits culturels africains contemporains constituent à ce titre des réponses hybrides et complexes à l'histoire (particulièrement coloniale) et au contexte mondial actuel. Cela peut décevoir les défenseurs occidentaux et africains d'une Afrique « authentique » retournant vers ses racines, mais c'est la réalité qu'il convient d'accepter et sur laquelle il faut travailler aujourd'hui. Un appel, en somme : Ezra Chitando s'adresse directement aux chercheurs, les engageant à travailler sur cette Afrique dont il fait état, dans une démarche pluridisciplinaire, qui permet d'aborder et de comprendre toute la complexité de l'Afrique contemporaine par le biais de ses produits culturels.

Au total, il s'agit, d'une part, d'un document intéressant sur le «phénomène gospel » au Zimbabwe, dans un pays sur lequel peu de chercheurs se penchent (ou alors pour y étudier les aspects traditionnels de ses cultures), et qui vit aujourd'hui une période difficile mais charnière pour son histoire, et riche de productions culturelles et artistiques diverses. D'autre part, on trouve là une base de réflexion sur la manière avec laquelle les sciences humaines se doivent de s'arrêter sur les cultures contemporaines africaines, et plus généralement sur son(ses) visage(s) actuel(s), et poussant à prendre en compte la réalité concrète de ce continent, à se départir des rêveries d' " authenticité » et de «pureté culturelle » qui président souvent, même dans les cercles de recherche universitaire, à son appréciation.

Véronique LiOT

Falceto, Francis. - Abyssinie Swing. A Pictorial History of Modern Ethiopian Music. Images de la musique éthiopienne moderne. Addis Ababa, Shama Books, 2002, 120 p., photos, bibl.

Par la frivolité et l'exubérance qu'elles révèlent, les images des scènes musicales éthiopiennes renversent les clichés misérabilistes qui collent habituellement à ce pays. Par cette histoire iconographique des musiques modernes 
d'Éthiopie, Francis Falceto présente une nouvelle facette d'un patrimoine qu'il a déjà substantiellement réhabilité en publiant la collection de disques éthiopiques/ ityophiks ${ }^{1}$, où sont compilés les tubes du groove éthiopien des années 1950 à 1970. À leur écoute, même les oreilles les plus blasées ont la surprise de découvrir un style musical idiosyncrasique, fait de combinaisons harmoniques, modales et rythmiques étonnantes. Outre la volonté de consolider les ressources de ce répertoire méconnu face à la voracité uniformisatrice des productions les plus fâcheuses de la World Music, l'auteur cherche surtout à fournir aux Éthiopiens les moyens de se réapproprier les expériences les plus réussies de leur participation à la modernité. Ce projet est mené à bien, d'abord par les qualités éditoriales de ce livre bilingue — anglais et français —, judicieusement présenté par une mise en page véritablement «swinguante ». Le rythme qu'elle produit apporte en effet un plaisir de lecture qui mérite d'être souligné.

114 documents photographiques, inédits pour une bonne part, sinon tirés de sources peu fréquentées, sont ici présentés. Après les premières images photographiques de musiciens éthiopiens traditionnels prises par des explorateurs européens, on voit apparaître sous le règne de Menilek II (1889-1913) des instruments et des mélodies des fanfares européennes qui furent intégrés au protocole notamment à l'occasion des grands banquets royaux. Pour moderniser son royaume, le seul État en Afrique resté indépendant, ce souverain accueillit en effet des innovations de toutes sortes. Cette musique officielle se constitua véritablement au milieu des années 1920, quand le ras Täfäri (futur roi des rois Haylä Sellasié) recruta à Jérusalem un orchestre d'orphelins arméniens, rescapés du génocide de 1915. Cette fanfare dissolue au terme d'un contrat de quatre ans, fit école et permit la multiplication de formations institutionnelles préférant les timbres cuivrés aux dépens de l'ambition jamais réalisée de la création d'un orchestre symphonique national. La période d'occupation italienne (1936-1941) fut marquée par la répression à l'encontre des azmari, chansonniers, dont l'art subtil du double sens représentait un potentiel contestataire inadmissible pour un régime totalitaire. À la Libération, c'est par l'Érythrée, dans une situation de semi-décolonisation propice à l'effervescence sociale et créatrice, que s'ouvrit la porte aux influences électriques et écléctiques afro-américaines, qui ne cessèrent de s'amplifier et de nourrir l'originalité des pionniers du Swinging Addis.

Parmi la galerie de portraits qui conclut l'ouvrage, consacrée aux figures de proue, arrêtons-nous par exemple à Getachew Mekurya (pp. 94-95), inventeur du jeu de saxophone shelläla, c'est-à-dire une adaptation des chants de bravoure par des solos de sax rugissants. Sa prestation en tenue de guerrier, coiffé d'une crinière de lion évoque des résonances avec Menelik (Lion of Judah), un des thèmes de jungle les plus stupéfiants enregistrés par Duke Ellington ${ }^{2}$, composé en 1941 par le trompettiste Rex Stewart, s'ouvrant sur des borborygmes produits dans l'embouchure, accompagnés par une rythmique endiablée.

Au fil des pages, le bouillonnement des sons et la frénésie des corps semblent aller crescendo, à mesure que le régime du vieux monarque s'ankylose dans un absolutisme paternaliste. Le repli des illusions modernisatrices de ce régime dans le microcosme urbain d'Addis Abäba a favorisé l'éclosion d'une

1. $11 \mathrm{CD}$ parus chez Buda Musique, Paris.

2. Duke Ellington s'est produit à Addis Abäba le 22 novembre 1973, comme en témoigne la photo 112, le montrant en compagnie de Mulatu Astatqé, arrangeur de génie. 
bulle d'insouciance et de cosmopolitisme. L'épopée s'arrête abruptement en 1974, quand les aspirations d'un mouvement social de grande ampleur furent confisquées par une junte militaire instituant un régime révolutionnaire stalinien. L'auteur insiste sur l'oubli total de ce répertoire opéré par la censure, la répression et l'exil. Selon lui, il ne reste rien aujourd'hui de cet âge d'or. Ce point de vue est probablement exagéré. Certains de ces musiciens sont restés très populaires et prolifiques malgré le laminage idéologique et propagandiste imposé à leur art. Depuis la chute de la junte militaire en 1991, et l'adoption d'un modèle économique libéral, l'importation de synthétiseurs et de boîtes à rythmes a indéniablement affadi la production musicale contemporaine. On peut y observer cependant une continuité des standards et les bourgeonnements d'une inventivité nouvelle.

Éloi FIQUET

Leymarie, Isabelle. - Cuban Fire. Musiques populaires d'expression cubaine. Paris, Éditions Outre Mesure, 1997, 351 p., index, bibl. («Contrepoints »).

Ce livre d'Isabelle Leymarie s'inscrit dans la continuité de plusieurs autres publications de l'auteure sur les musiques latino-américaines en général et sur la salsa et le Latin Jazz en particulier. Elle décrit ici de façon très fouillée l'émergence de différents styles musicaux cubains et l'histoire de leurs fusions avec d'autres styles caribéens et nord-américains. L'objectif est ambitieux puisqu'il s'agit de couvrir à la fois toute la période qui va du début du $\mathrm{Xx}^{\mathrm{e}}$ siècle à nos jours et plusieurs lieux-clés de création et de diffusion de ces musiques, parmi lesquels essentiellement La Havane, New York et Porto Rico.

Pianiste et musicologue, I. Leymarie est passionnée par son sujet, et elle en est même partie prenante. Elle n'hésite donc pas, dès la première phrase, à porter des jugements de valeur (la musique afro-cubaine serait ainsi « l'une des plus riches et des plus séduisantes du monde », p. 9) et à opérer des choix et des sélections qui ne seront évidemment pas du goût des lecteurs d'opinion différente. «Si, sur le plan de la créativité, la musique de danse cubaine - et la salsa en particulier - paraît traverser une période de relative stagnation due à ses limitations harmoniques, le Latin Jazz en revanche ne cesse d'évoluer », nous dit-elle en conclusion, après s'être appliquée à démontrer au fil du texte la richesse du Latin Jazz, son style de prédilection.

On pourra donc se faire l'écho des critiques et commentaires que suscitent les livres d'I. Leymarie dans le milieu des spécialistes du genre (artistes et amateurs), polémiques qui concernent souvent la paternité des styles et des morceaux, l'antériorité d'un style par rapport à un autre (le mambo est-il un dérivé du cha-cha-cha, ou bien est-ce le contraire, comme l'affirme l'auteure ?) ou la façon d'établir des typologies. À ce titre, l'idée du tableau synoptique ${ }^{3}$ (p. 312) est en elle-même très contestable. Pourquoi présenter de façon aussi tranchée les filiations entre styles musicaux, alors que tout le livre montre bien leur perméabilité ? Il aurait été plus intéressant d'expliquer pourquoi et comment certains artistes souhaitent, dans des contextes précis, se démarquer

3. Idée qu'avaient déjà eue M. Faligand et D. Chatelain dans Percussions, 44 : 26-28. 
des autres en donnant un nom à leur façon de faire de la musique. C'est par exemple le cas du terme rumba brava, qui n'est absolument jamais employé à Cuba, ou du genre songo (terme finalement peu usité aujourd'hui hormis par le groupe Los Van Van) qui prétendait au début des années 1990 distinguer les orchestres cubains des orchestres de salsa, tout en soulignant leur modernité par rapport au son.

Le lecteur passionné sera en revanche comblé par la perspective encyclopédiste du texte, qui ne lui épargne aucun détail sur les noms et les surnoms des protagonistes, leurs filiations orchestrales, leur biographie, leurs initiatives, le tout relayé par un index extrêmement bien conçu. Certains ne manqueront pas de s'étonner du décalage entre les parties, reflet des goûts (et des limites) de l'auteure, qui apparemment ne s'intéresse ni à la musique populaire cubaine récente (Los Van Van est accusé de sombrer parfois dans la vulgarité p. 229 et la Nueva trova est qualifiée de «mièvre et bien pensante» p. 147), ni à l'infortuné Oscar D'León à qui elle ne daigne consacrer que 4 lignes, pour ne citer que quelques exemples. Parfois fastidieuses (les patronymes latinoaméricains ne sont pas très variés), ces descriptions minutieuses ont toutefois le mérite de rendre très bien compte des interactions et des échanges, souvent méconnus, opérés entre Cuba, Porto Rico et les États-Unis, mais aussi le Mexique, la France et la République Dominicaine entre autres. Elles témoignent également de l'intense circulation des artistes de formation en formation, phénomène qui empêche de typologiser styles et orchestres de façon stricte.

Les parties qui concernent les États-Unis sont sans doute les plus abouties : l'auteure apporte un éclairage intéressant sur le contexte politique, social et économique des processus qu'elle décrit, en s'appuyant sur les témoignages des artistes eux-mêmes. Elle décrit le rapprochement entre les musiciens cubains, portoricains et noirs-américains dans les années 1940 et 1950, au moment où ils subissent un même racisme. Plus loin on comprend cependant que les échanges musicaux ne présument en rien d'une véritable solidarité entre les groupes, notamment après la première grande vague d'immigration cubaine des années 1960. Les exilés sud-américains répugnent en effet à être assimilés à des «Noirs », et si la salsa naît dans les années 1970, ce n'est pas un hasard : elle « confère aux Latinos un sentiment de fierté et d'identité culturelle » (p. 239). I. Leymarie aurait pu développer l'analyse en s'interrogeant sur la notion de raza latina, scandée depuis cette époque dans de nombreux morceaux, notion qui ne présume en rien à son tour de l'émergence d'un véritable mouvement communautaire, les artistes se livrant en réalité une concurrence acharnée qui contribue à les maintenir dans une situation d'exploités (p. 301).

Ces remarques fines tranchent avec les parties consacrées à Cuba où l'auteure plaque un certain nombre de lieux communs sans chercher à analyser leur contexte de production. Les apports africains à la musique populaire cubaine sont par exemple soulignés et valorisés, et l'accent est mis sur les tambours batá et la «tradition yoruba» au détriment d'autres influences («les Bantous, plus rapidement assimilés au substrat blanc que d'autres groupes africains, perdirent rapidement certaines traditions religieuses » p. 19). Ce qui est ici présenté comme une évidence relève en réalité d'une construction, dans laquelle les institutions commerciales et nationales, les intellectuels cubains liés au mouvement afro-cubaniste et les luttes de pouvoir entre musiciens ont joué un rôle déterminant. 
Il sera peut-être plus intéressant de consulter cet ouvrage pour ce qu'il est, c'est-à-dire une façon parmi d'autres de présenter l'histoire de ces musiques, et de le comparer avec les nombreuses publications qui ont récemment vu le jour sur ce sujet. Malheureusement l'auteure néglige la plupart du temps de citer ses sources en cours de texte, ce qui rend l'analyse critique difficile. Signalons toutefois quelques courts entretiens avec des musiciens transcrits en fin d'ouvrage, riches d'informations sur les modes d'appréciations et les enjeux de la catégorisation.

\section{Kali ARgYRIADIS}

Martin, Denis-Constant \& RouefF, Olivier. - La France du jazz. Musique, modernité et identité dans la première moitié $d u X X^{e}$ siècle. Marseille, Éditions Parenthèses, 2002, 323 p., index (« eupalinos »).

C'est à la fin de la Première Guerre mondiale que la France découvrit le jazz. Les tournées des fanfares de soldats noirs américains propagèrent avec succès les surprenantes musiques élaborées Outre-Atlantique. Durant les années folles de l'Entre-deux-guerres, cette nouvelle mode gagna le devant de la scène. Les jazz bands commencèrent par séduire l'avant-garde artistique et intellectuelle, puis leurs rythmes syncopés ont peu à peu imbibé le répertoire des musiques populaires. Les jeunes amateurs de jazz attendaient de cette musique qu'elle décape les décombres de la vieille Europe. Pour régénérer une civilisation qu'ils jugeaient décatie, ils croyaient dans les vertus de ce nouvel art qui conjuguait les pulsations frénétiques de la modernité américaine et la spontanéité primitive attribuée à ses origines africaines. L'invention d'un jazz français fut d'abord hésitante, vécue par ses hérauts comme une pâle imitation du modèle noir américain. Sous l'Occupation, ce jazz national commença à rencontrer un vaste public en se développant dans les étroits interstices aménagés par les ambiguïtés des catégorisations nationales et raciales qui avaient prévalu dans sa réception, et en servant d'étendard à la contre-culture zazou. Le retour du jazz américain fut ensuite célébré comme la musique enthousiasmante de la Libération. L'importation des premiers enregistrements de be-bop provoqua alors de violentes polémiques entre les partisans du «vrai jazz », institué comme authentique lors des trois décennies précédentes, et les amateurs de nouveautés, ouverts aux infinies possibilités créatrices pouvant s'exprimer dans ce langage musical. C'est au seuil de ce changement d'époque que ce livre s'arrête.

Cette nouvelle histoire des débuts du jazz en France s'ajoute à d'autres ouvrages récemment parus sur cette question ${ }^{4}$, participant de l'évaluation d'un siècle (approximativement) d'expansion, de diversification et de standardisation de cet univers musical. Cette entreprise procède aussi du développement des outils d'analyse sur les contextes sociaux de production, de réception et de diffusion du champ musical. Dans le domaine du jazz, qui a été assez tardivement investi par la recherche universitaire, la caractéristique notable de l'étude

4. Ludovic Tournès, New Orleans sur Seine. Histoire du jazz en France, Paris, Fayard, 1999. Jean-Dominique BRIERRE, Le Jazz français de 1900 à aujourd'hui, Paris, Éditions Hors Collection, 2000. Signalons aussi le numéro spécial de L'Homme, 2001, $\mathrm{n}^{\circ}$ 158-159, sur le thème « Jazz et Anthropologie ». 
de Denis-Constant Martin et Olivier Roueff est la polyphonie du propos correspondant à la qualité première de l'objet qu'il décrit. En proposant deux textes traitant de la même période à partir de sources différentes, les auteurs exposent des variations sur une même grille, ce qu'on nomme en jazz des chorus. Les solistes s'y illustrent avec brio, leurs analyses s'éclairant mutuellement malgré quelques redondances inévitables.

Le texte de Martin se fonde sur une approche prosopographique des musiciens et des amateurs de jazz, qui ont été les inventeurs - au sens double de découvreurs et de créateurs - du jazz en France. Les témoignages de vétérans, notamment du Hot Club de France (créé en 1932), sont apportés par de larges citations surtout extraites des deux principaux magazines de jazz en France, Jazz Hot et Jazz Magazine. En contrepoint, Roueff propose une étude des discours sur la réception du jazz à travers la presse des années 1920 et 1930. Il y observe finement la constitution d'une critique contribuant, par ses controverses et ses convictions, à définir le jazz comme un genre musical autonome c'est-à-dire doté d'une corporéité propre réalisée par diverses incarnations institutionnelles, organologiques, sensuelles, et langagières. Pour suivre ces développements le lecteur n'est pas renvoyé à d'inaccessibles références, mais une sélection des sources les plus parlantes est fournie dans de copieuses annexes, rassemblant 45 textes. Ce recueil d'engouements, de questionnements, d'incompréhensions et de débats montre que les problèmes qui continuent de tirailler la critique de jazz ont très tôt été posés avec acuité.

Il ressort de ce triple ouvrage une ritournelle obsédante qui a accompagné la réception du jazz en France, fonctionnant sur l'ambivalence entre deux pôles d'altérité, l'Amérique et l'Afrique. Les étincelles jaillies des frictions musicales qui se sont exercées entre ces deux continents ont produit des embrasements créatifs dont la propagation fut captée avec crainte et émerveillement en Europe. Ces appréciations contradictoires sont illustrées par une longue série de dualismes à travers lesquels ont été pensés les traits constitutifs du jazz : la mécanisation industrielle et la sauvagerie primitive ; l'arrangement et la spontanéité ; l'écriture des orchestrations (style dit straight) et l'oralité des improvisations (style dit hot) ; la musique sérieuse et le divertissement... De telles tensions catégorielles ont été identifiées au sein même de la musique par le «conflit entre le rythme et la mesure $»^{5}$, formule due à André Schaefner plus tard reprise pour expliquer la source mystérieuse du swing.

Le fil conducteur de ces observations est de considérer les discours sur le jazz comme un « langage utile pour parler d'autre chose » (p. 13), c'est-à-dire révélateur des incertitudes et des mutations de l'identité française face aux expériences de l'exotisme colonial et de l'accélération de la révolution industrielle. Ces problèmes ont stimulé d'autres démarches artistiques et intellectuelles. Martin ne manque pas ainsi de souligner les parallèles et les trajectoires transversales entre la découverte du jazz en France et la mise au point de l'anthropologie africaniste. Une fois posés, ces arguments devraient servir à nourrir une perspective comparative élargie à l'Europe aux autres continents, permettant d'évaluer les répercussions différentielles du jazz à travers le monde.

Éloi FIQUET

5. André Schaeffner \& André Ceeuroy, Le jazz, Paris, J.-M. Place, 1988 : 35. 
Palmberg, Mai \& KirkegaArd, Annemette (eds). - Playing with Identities in Contemporary Music in Africa. Uppsala, Nordiska Afrikainstitutet, 2002, 182 p.

Ce livre, essentiellement rédigé par des spécialistes de musiques et sociétés africaines provenant de différentes disciplines, est plutôt de nature académique. Le politique et l'identité sont les deux principaux thèmes abordés, même si le concept d'authenticité, dans le sens critique du terme, y tient une place importante. Structuré de manière à diriger le lecteur vers une compréhension globale du sujet, ce livre s'adresse à un auditoire ciblé que je qualifierai de « scholars ». Et ce n'est pas surprenant car ce collectif a été créé à partir de textes et de séminaires présentés dans le cadre d'un congrès organisé par le projet de recherche «Cultural Images in and of Africa » du Nordic Africa Institute, du département «Musicology/Sibelius Museum» et du Centre for Continuing Education.

Le thème de ce congrès étant directement relié à l'identité, les conférenciers tentent, par le biais de diverses chroniques disciplinaires, de faire connaître au lecteur une partie de ce très vaste continent. Cependant, j'aimerais que le lecteur porte une attention toute particulière à l'introduction de cet ouvrage collectif. Écrit par Annemette Kirkegaard, et échelonné sur quelques pages seulement, ce texte fait un bon tour d'horizon de la question musicologie-ethnomusicologie, de la musique et de l'identité, de la World Music et du concept d'authenticité que j'ai cité précédemment.

Nous pouvons, avec ce collectif, déceler deux livres en un seul. Il y a cette espèce de retenue intellectuelle qui dérange mais qui, simultanément, peut être nécessaire si on veut bien comprendre tous les aspects de la musique comme moyen de véhicule des valeurs nationalistes et revendicatrices des peuples africains. Mais il y a aussi cet amour de la musique qui ressurgit dans le texte. Je pense à l'article de Simon Akindes qui laisse parler la fugue et la violence du peuple de la Côte-d'Ivoire. Un de ses chapitres, "Bloody conflicts », le démontre bien.

J'ai écrit, dans ma critique concernant le livre de Franck Tenaille (Le swing du caméléon), que le langage musical est parfois pauvre et difficile chez les auteurs qui veulent écrire au sujet de la musique. Étant donné que le défi est de taille pour quiconque veut le faire, je pense qu'il serait important de vous proposer le texte de Christopher Waterman qui lui, contrairement à bien d'autres, semble posséder cette qualité. À titre d'exemple, en voici un extrait: " Their imaginative manipulations of texture and timbre, tune and tone, speech and bodily practices (dance, costuming) can create imaginative "quality spaces". » Son texte "Big Man, Black President, Masked One : Models of the Celebrity Self in Yoruba Popular Music in Nigeria », nous fait découvrir ce que le concept d'identité, vécu par l'intermédiaire d'acteurs importants de la musique africaine tels Fela Anikulapo-Kuti, Alhaji Barrister et King Sunny Ade, veut réellement dire. Waterman est un auteur tout à fait remarquable qui sait trouver les bons mots pour parler de la musique, lui donner un langage. Cette sensibilité vient non seulement de sa formation de musicien, mais également de son expérience de pionnier dans l'articulation d'une réflexion à la fois ethnographique et critique au sujet de la production culturelle en Afrique contemporaine. À mon avis, Christopher Waterman est le doyen dans ce domaine.

Enfin, ce livre peut être lu de différentes façons. Selon l'intérêt du lecteur, il prendra une texture différente et une dimension personnelle. Cependant, la 
caractéristique fondamentale de ce livre réside dans la qualité inégale de ses contributions : mais c'est parfois l'inconvénient des ouvrages collectifs !

Marcel SAVARD

SIron, Jacques. - Dictionnaire des mots de la musique. Paris, Éditions Outre Mesure, 2002, 305 p., index, bibl.

Le dictionnaire des mots de la musique que vient de publier Jacques Siron aux Éditions Outre Mesure, nous offre un passionnant panorama des traditions musicales, des noms d'instruments de tous pays, des termes techniques — des plus anciens aux plus récents - et historiques, des styles musicaux, des appellations familières utilisées dans le jargon des musiciens, des genres (jazz, rock, classique, musiques du monde, rap, musques traditionnelles), de l'informatique musicale, du son.... Ce dictionnaire représente une source d'informations remarquable en quatre langues (français, italien, anglais, allemand).

LA RÉDACTION

TenAILle, Franck. - Le swing du caméléon. Musiques et chansons africaines, 1950-2000. Arles, Actes Sud, 2000, 315 p.

Avec ce livre, Frank Tenaille nous propose une anthologie de la musique africaine échelonnée sur plusieurs décennies. Même s'il est parfois difficile de parler de la musique africaine sans se préoccuper de l'environnement social, politique et culturel, Franck Tenaille, transporté par un style littéraire rappelant plutôt la chronique, et assurément amoureux de musique africaine, réussit à sortir du contexte géopolitique africain les artistes qui ont fait bouger tout un continent. Et dans ce sens, les personnages centraux de ce livre ne sont plus seulement des ambianceurs politisés ou persécutés, mais aussi des hommes et des femmes qui ont un parcours de vie extraordinairement riche. Autrement dit, derrière le personnage public souvent connu, ou reconnu pour son parcours musical original et parfois même «virginal », il y a une histoire qui nous touche et qui nous propose une nouvelle façon d' « entendre » l'Autre.

Ce genre littéraire suggère au lecteur une certaine intimité, une proximité émotive avec les acteurs du livre qui chantent l'Afrique, c'est-à-dire celle qui est unie par le son et la parole de ses bâtisseurs.

La structure du voyage culturel qui est proposée par l'auteur se répartit en différentes étapes historiques suggérant des périodes de bouleversement importantes telles les indépendances, représentées ici par un Joseph Kabaselé qui composera Indépendance cha-cha. Evidemment, Tabu-Ley Rocheleau qui, selon Tenaille, est l'héritier stylistique de Kabaselé, rappellera au lecteur l' "épisode » Mobutu et l'exil politique obligé de certains artistes. Cette période d'ostracisme sera d'ailleurs maintes fois évoquée dans ce livre. Mais comme le démontre l'auteur, l'exil ne sera pas seulement à sens unique. Parce que la quête d'identité, ou plutôt l'affirmation de son identité, sera aussi vécue à l'extérieur de l'Afrique, Franck Tenaille nous fera voyager avec les artistes et les idéaux de ces représentants du terroir africain, de ces diplomates chantants. 
La «persécution»sera donc, elle aussi, sans frontière. Le bagage culturel transporté par ces troubadours sera inévitablement métissé, influencé par une culture elle-même déjà transformée. Ici, le paradoxe d'une culture occidentale en mal d'exotisme nous fera revivre le tragique destin de plusieurs artistes qui étaient venus s'exprimer librement, en tout cas le pensaient-ils. Je pense à Myriam Makeba qui, entre le silence et l'exil, ou, entre les Black Panthers et la résiliation de ses contrats discographiques, dût retourner en Afrique parce que constamment traquée par la force policière. En parlant de Makeba, Tenaille écrit : « [...] La realpolitik prendra le pas sur les grands sentiments humanistes qui ont accompagné son arrivée aux États-Unis. »En somme, les périodes de quarantaine imposées par les autres, qu'elles soient africaines ou occidentales, ne représentent que le pâle reflet d'une époque que l'on peut qualifier de « réductrice » pour le genre humain, pire encore, de marchande d'idées corrompues. Évidemment, dans un tel contexte, comme le souligne Tenaille, c'est la voix des autres qui doit subir le sort de tous. Par conséquent, les musiciens sont devenus simultanément des alliers et des ennemis.

Parallèlement, nous pouvons, par la représentation historique des événements qui nous est suggérée, suivre le parcours de ceux qui ont pensé s'approprier l'Afrique. Certes, ils y ont laissé leurs odeurs, leurs couleurs et souvent leurs drapeaux, mais jamais ils n'ont pu conquérir l'esprit, l'ancienneté, le modus operandi, le langage et l'intention de ses musiciens. Dans ce sens, plutôt que de métisser son âme, la musique africaine, dans son ensemble, s'est plutôt inspirée de ces contacts.

Il y a cependant un bémol au livre de Franck Tenaille. Il faut être prudent dans la lecture du texte car l'auteur relie constamment musique, politique et identité, et cela, sans vraiment nous entretenir sur une autre réalité musicale et culturelle importante, c'est-à-dire l'authenticité, la musique traditionnelle. Même s'il est question de métissage, ou d'emprunt culturel réalisé au fil des contacts plus ou moins importants et prolongés des colonisateurs et des habitants de ce continent, il ne faut pas oublier que la musique traditionnelle pose les fondements même d'une musique nationale élargie, identitaire. D'ailleurs, ce besoin viscéral d'authenticité se retrouve au cœur même des revendications nationalistes des peuples africains. Ici, la musique rappelle que l'identité n'est pas un but en soi, mais plutôt une façon de voyager, de vivre.

Depuis plusieurs années, nous sommes submergés d'écrits plus ou moins adaptés au sujet de la musique populaire et nationaliste. Le langage est souvent pauvre et difficile à entendre. Dans ce sens, je pense que le langage musical, pour un grand nombre de chroniqueurs qui s'intéressent à la musique, est devenu un grand défi. Comment décrire l'intention de l'artiste lorsqu'il pense la création de sa pièce musicale, comment décrire un son que l'émotion transporte dans tout son corps, voilà, à mon avis, le grand défi de celui qui veut écrire « la musique ».

Tenaille nous propose, en fin de parcours de son livre, des annexes qui viennent parfaire notre connaissance de l'environnement musical de ce continent. Par exemple, les aires musicales, les genres et styles musicaux et un glossaire des instruments qui nous fait voyager et sentir le bois de manguier tellement il est précis.

Enfin, ce livre s'adresse surtout à tous ceux et celles qui désirent connaître l'Afrique à travers sa musique. Nonobstant les conflits politiques internes et internationaux qu'a pu vivre ce continent, vous découvrirez, dans les propos de Franck Tenaille, les acteurs de la renaissance et de la continuité africaine.

Marcel SAVARD 